\title{
Nucleation and Growth of Crystalline Grains in RF-Sputtered $\mathrm{TiO}_{2}$ Films
}

\author{
J. C. Johnson, ${ }^{1}$ S. P. Ahrenkiel, ${ }^{2}$ P. Dutta, ${ }^{3}$ and V. R. Bommisetty ${ }^{3}$ \\ ${ }^{1}$ Physics Department, South Dakota School of Mines \& Technology, Rapid City, SD 57701, USA \\ ${ }^{2}$ Nanoscience and Nanoengineering Department, South Dakota School of Mines \& Technology, Rapid City, SD 57701, USA \\ ${ }^{3}$ Electrical Engineering Department, South Dakota State University, Brookings, SD 57007, USA
}

Correspondence should be addressed to S. P. Ahrenkiel, phil.ahrenkiel@sdsmt.edu

Received 3 June 2009; Accepted 25 July 2009

Recommended by Xiaowei Sun

Amorphous $\mathrm{TiO}_{2}$ thin films were radio frequency sputtered onto siliconmonoxide and carbon support films on molybdenum transmission electron microscope (TEM) grids and observed during in situ annealing in a TEM heating stage at $250^{\circ} \mathrm{C}$. The evolution of crystallization is consistent with a classical model of homogeneous nucleation and isotropic grain growth. The twodimensional grain morphology of the TEM foil allowed straightforward recognition of amorphous and crystallized regions of the films, for measurement of crystalline volume fraction and grain number density. By assuming that the kinetic parameters remain constant beyond the onset of crystallization, the final average grain size was computed, using an analytical extrapolation to the fully crystallized state. Electron diffraction reveals a predominance of the anatase crystallographic phase.

Copyright (c) 2009 J. C. Johnson et al. This is an open access article distributed under the Creative Commons Attribution License, which permits unrestricted use, distribution, and reproduction in any medium, provided the original work is properly cited.

\section{Introduction}

The broad range of industrial and technological applications for the wide-gap semiconductor $\mathrm{TiO}_{2}$ has grown to include numerous roles in energy conversion and storage, particularly relating to photovoltaics [1] and photocatalysis [2]. Along with its useful electrical and optical properties [3], $\mathrm{TiO}_{2}$ provides the benefits of low toxicity, good chemical stability, and ease of fabrication [4]. As-deposited, amorphous-titania thin films are commonly produced at low temperature by a range of methods, commonly spincoating of sol-gel precursors [5] and physical vapor deposition [6]. Postdeposition crystallization provides additional influence on crystallographic phase and the promotion of desirable microstructural characteristics, with potential relevance toward developing new, low-cost methods of synthesis, while also permitting fundamental studies of materials properties.

Under ambient conditions, kinetic barriers typically prevent the rapid phase transformation from a metastable, amorphous microstructure to a crystallized, solid state [7]. Mechanisms leading to crystallization may include spon- taneous grain nucleation and grain growth, which can be thermally activated at elevated temperatures, using thermal annealing $[8,9]$ or electron-beam irradiation [10-12]. This work aims to shed light on the kinetics of nucleation and growth crystalline grains in as-deposited amorphous, RFsputtered $\mathrm{TiO}_{2}$ films, during isothermal annealing in a TEM heating stage. Several experiments have examined crystallization during thermal annealing of sol-gel $\mathrm{TiO}_{2}$ films $[8,9,12-$ 14], which may be driven by the coalescence of nanosized amorphous nuclei. Metallurgical phase transformations are more often associated with growth of individual grains into the surrounding amorphous matrix [15], leading to a poly- or microcrystalline grain structure. The data presented here are analyzed from the perspective of classical phasetransformation theory, assuming homogenous nucleation and isotropic grain growth. The final, average grain size in these films is predicted by analytical extrapolation of the modeled behavior to the fully crystallized state. The goal is to understand and influence the crystallization process, providing for optimization of material quality and improvements in the performance of $\mathrm{TiO}_{2}$ films for device applications. 


\section{Experiment}

$\mathrm{TiO}_{2}$ was radio-frequency sputtered onto silicon-monoxide coated and carboncoated, 200-mesh Mo TEM grids, obtained from Electron Microscopy Sciences. A steel TEM grid holder was used to suspend the grids in the reactor in an inverted orientation, with contact only to the grid periphery. The thickness of the support films is specified by the manufacturer to be in the range of $15-30 \mathrm{~nm}$ for silicon monoxide and 20-30 nm for carbon. A $2^{\prime \prime}$-diameter $\mathrm{TiO}_{2}$ target with $99.9 \%$ purity from Kurt J. Lesker Company was sputtered with a process pressure of 5 mTorr, plasma power of $150 \mathrm{~W}$, deposition rate of $0.02 \mathrm{~nm} / \mathrm{s}$, Ar gas flow rate of $20 \mathrm{sccm}$, and a nominal thickness of $h=100 \mathrm{~nm}$. To reduce crystallization during deposition, no substrate heating was used. However, we note the presence, prior to postdeposition treatment, of small crystalline nuclei, representing less than $1 \%$ of the total sample volume.

The samples were imaged on a Hitachi H7000-FA TEM, operated at $125 \mathrm{KV}$, using an Olympus SIS Veleta 14-bit, 4-megapixel CCD side-mount camera. The Olympus SIS iTEM imaging software was used to acquire the image series, by automated acquisition at 2-second intervals. In situ annealing was accomplished with a Gatan Model 628 single-tilt heating holder. The heating stage uses a tantalum furnace, capable of temperatures over $1000^{\circ} \mathrm{C}$, with a hexring retaining screw to secure the sample. To reach a final dwell temperature of $250^{\circ} \mathrm{C}$, the heating stage was ramped up initially to $100^{\circ} \mathrm{C}$, then $200^{\circ} \mathrm{C}$, and allowed to dwell at each temperature for approximately 1-2 minutes to reduce drift. Enhanced crystallization in the analyzed region was sometimes observed, presumably due to local heating by the electron beam. TEM image series were taken in conical dark-field mode, using beam precession about the optic axis at $20 \mathrm{~Hz}$, with an objective aperture of diameter $3.3 \mathrm{~nm}^{-1}$ placed in the back focal plane of the objective lens to increase contrast between amorphous and crystalline regions. The beam tilt was adjusted so that the anticipated position of the first strong diffraction ring was approximately centered on the optic axis.

A subset of images acquired 20 seconds apart was selected for quantitative analysis. Using Adobe Photoshop Elements 2.0, a portion of each image was cropped from a common area. Automatic and manual edge recognition tools were used to differentiate crystalline from amorphous regions. The segmented images were then imported to Gatan Digital Micrograph to determine the crystalline volume fraction $X_{c}=V_{c} / V$, where $V_{c}$ and $V$ are the crystallized and total volumes, respectively, which were assumed to be proportional to the projected areas. Direct grain counting was performed using an elliptical annotation tool to demarcate previously counted grains, to determine the areal grain number density $\rho_{g}=N_{g} / A$, where $N_{g}$ is the number of grains in the film area $A$. Errors in $X_{c}$ were taken as $4 \cdot X_{c} \cdot\left(1-X_{c}\right) \cdot(5 \%)$, where $5 \%$ was estimated to be the maximum random error in repeated measurements of $X_{c}$. The fractional error in $\rho_{g}$ was taken as $N_{g}{ }^{-1 / 2}$.

For cinematic visualization of the entire crystallization process, the contrast for all the photos was normalized, then
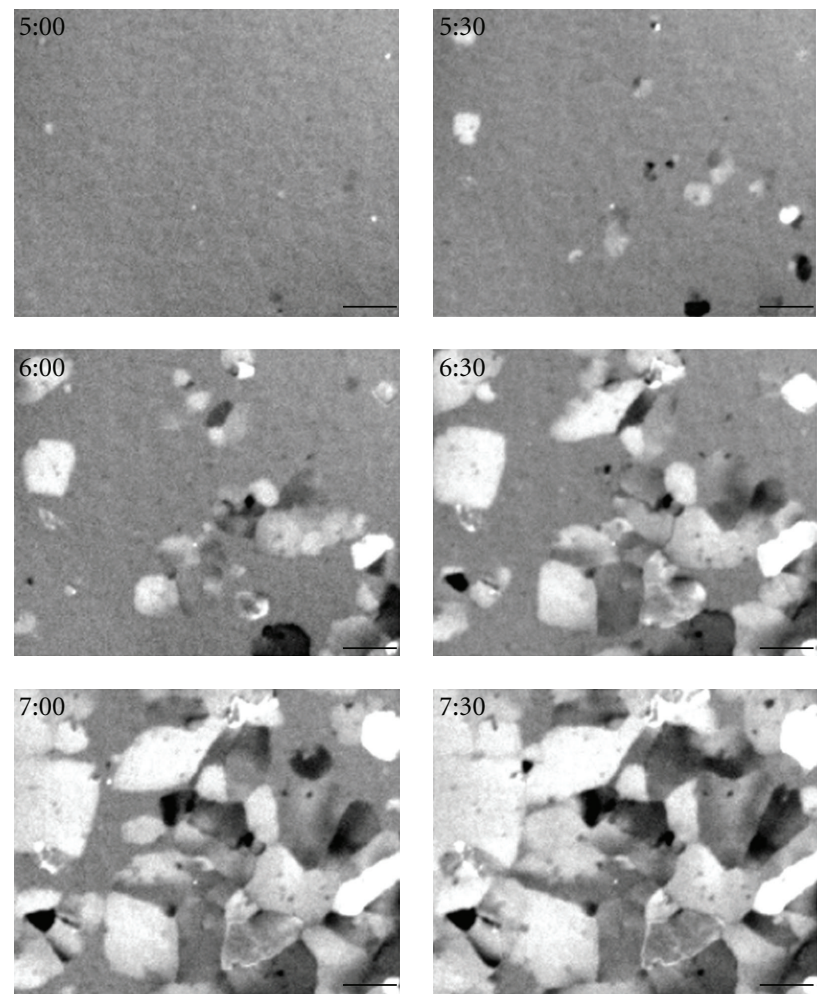

FIgURE 1: Aligned TEM images taken of a single area during crystallization. Time stamp is in min:sec. Images were acquired 30 seconds apart. Scale bar is $500 \mathrm{~nm}$.

the entire image series was aligned by a combination of crosscorrelation and iterative difference minimization about areas containing clearly identifiable features. The image series was then imported to Apple QuickTime to generate a video representation.

Selected-area electron diffraction patterns were acquired from circular areas several microns in diameter. Line patterns were then generated by rotational averaging in Digital Micrograph, allowing intensity to be plotted as a function of $1 / d$, where $d$ is the Bragg interplanar spacing.

\section{Results}

Our analysis assumes random grain nucleation within the amorphous matrix [16]. Lateral grain growth commences upon nucleation, proceeding until impingement of the grain onto other grains. In several repeated trials, we observed the onset of rapid grain nucleation within several seconds of reaching $250^{\circ} \mathrm{C}$. Nearly complete crystallization occurred within 2.5 minutes after the onset of nucleation (Figure 1).

In classical solid-state phase change theory, the variation of $X_{c}$ with $t$ during $2 \mathrm{D}$ grain growth is described by

$$
X_{c}(t)=1-\exp \left\{-\left[\frac{t-t_{0}}{t_{c}}\right]^{3}\right\},
$$

for $t \geq t_{0}$, where $t_{0}$ is the incubation time and $t_{c}$ is a characteristic crystallization time. 


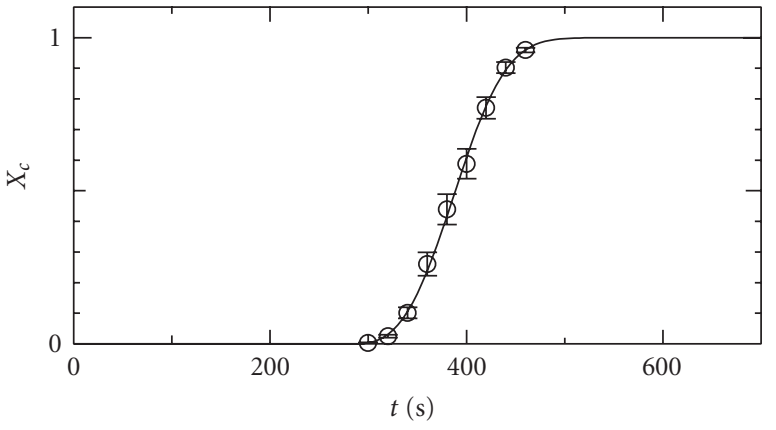

(a)

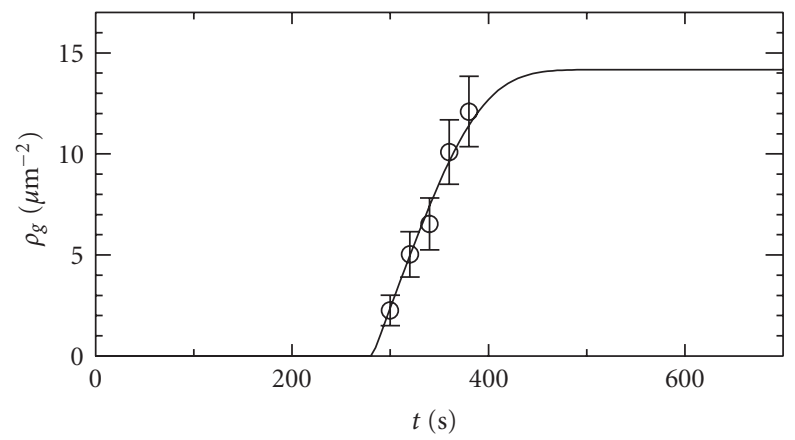

(b)

FIGURE 2: Crystallization parameters versus time from TEM data on $\mathrm{TiO}_{2}$ films annealed at $250^{\circ} \mathrm{C}$ : (a) $X_{c}(t)$; (b) $\rho_{g}(t)$. The curves were computed from simultaneous, multivariate leastsquares fits on both data sets.

Assuming a nucleation rate $r_{n}$, the variation of $\rho_{g}$ with $t$ for $t \geq t_{0}$ follows

$$
\rho_{g}(t)=\frac{\sqrt{\pi} r_{n} \cdot t_{c} \cdot h}{6} \cdot E_{3}\left[\frac{t-t_{0}}{t_{c}}\right]
$$

using the generalized error function:

$$
E_{3}(x)=\frac{3 !}{\sqrt{\pi}} \cdot \int_{u=0}^{x} e^{-u^{3}} d u .
$$

Leastsquares fitting of the measurements of $X_{c}(t)$ and $\rho_{g}(t)$ were combined into a single, multivariate data set, which was fit simultaneously to (1) and (2), by varying the parameters $t_{0}, t_{c}$, and $r_{n}$ (Figure 2).

The high quality of fit supports the validity of the applied model. The grain-growth velocity $s_{g}$ is computed from

$$
s_{g}=\left[\frac{3}{\pi \cdot r_{n} \cdot t_{c}^{3} \cdot h}\right]^{1 / 2} .
$$

The average grain size is estimated from the assumption of cylindrical grain shapes, having the cylinder axis normal to the film plane, which project to circular profiles in plan-view examination. Extrapolation to $t \rightarrow \infty$ gives the final, average grain size:

$$
\bar{d}_{g}(\infty)=1.20 \cdot\left(\frac{s_{g}}{r_{n} \cdot h}\right)^{1 / 3}
$$

TABle 1: Parameters for the crystallization at $250^{\circ} \mathrm{C}$ of $\mathrm{TiO}_{2}$ thin film: crystallization time $t_{c}$; nucleation rate $r_{n}$; grain growth velocity $s_{g}$; final average grain size $\bar{d}_{g}(\infty)$.

\begin{tabular}{lcc}
\hline Parameter & Value & Units \\
\hline$t_{c}$ & $120 \pm 4$ & $\mathrm{~s}$ \\
$r_{n}$ & $(1.31 \pm 0.11) \times 10^{-9}$ & $1 /\left(\mathrm{s} \cdot \mathrm{nm}^{3}\right)$ \\
$s_{g}$ & $2.03 \pm 0.09$ & $\mathrm{~nm} / \mathrm{s}$ \\
$\bar{d}_{g}(\infty)$ & $300 \pm 10$ & $\mathrm{~nm}$ \\
\hline
\end{tabular}

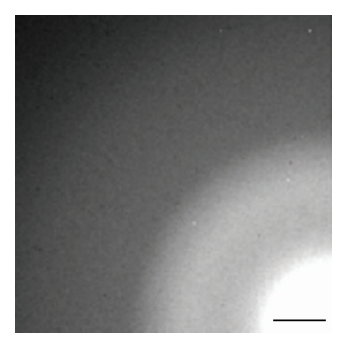

(a)

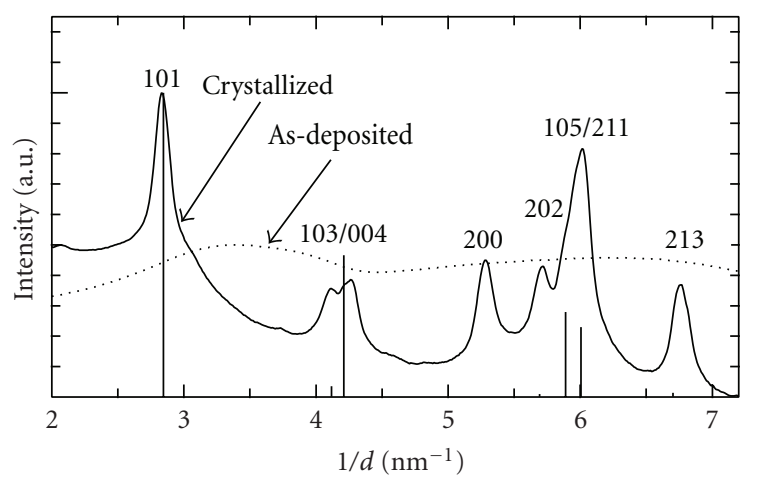

(c)
FIGURE 3: Selected-area electron diffraction data from $\mathrm{TiO}_{2}$ films: (a) as-deposited; (b) crystallized at $250^{\circ} \mathrm{C}$ (scale bar is $5 \mathrm{~nm}^{-1}$ ); (c) rotationally averaged line profiles. Bars are kinematical electrondiffraction simulation of anatase powder.

using $2 \cdot(3 / \pi)^{1 / 3} \cdot \sqrt{\Gamma(1 / 3)} \approx 1.20$. The columnar grain morphology assumed is applicable to larger grains (greater than $100 \mathrm{~nm}$ ), for which little overlap is evident in plan-view projection. However, the presence of smaller grains, which often overlap the larger grains in projection, may lead to a slight underestimate of the average, final grain size.

Calculated values of $r_{n}, s_{g}$, and $\bar{d}_{g(\infty)}$ are tabulated in Table 1. Based on the measurements of kinetic parameters at intermediate stages of crystallization, a value of $\bar{d}_{g}(\infty)=$ $0.30 \mu \mathrm{m}$ is predicted. This value is consistent with actual grain sizes seen in Figure 1, which range from tens of nm to nearly $1 \mu \mathrm{m}$.

The data set analyzed here was selected, based on the high image quality, from among several comparable data sets. However, the crystallization in all of the samples annealed under identical conditions transpired in a quantitatively similar fashion to that presented, resulting in comparable grain morphology and size. Note that each annealing treatment resulted in the simultaneous crystallization of the 
entire sample, though the area imaged by TEM was typically less than $25 \mu \mathrm{m}^{2}$, leaving a vast majority of each sample unobserved during transformation.

Diffraction patterns (Figure 3) clearly show the transition from the as-deposited, amorphous state to the final, crystallized state. The diffraction-ring positions of fully crystallized films are consistent with the anatase phase (space group $I 4_{1} /$ amd, no. 141), [17], based on a comparison with kinematic electron-diffraction simulations using EMS online software [18]. We note differences in relative intensities, particularly the presence of the kinematically forbidden 200 diffraction ring, which can arise from dynamical scattering, and may also be influenced by double diffraction, planardefect structure, and preferential orientation. Analysis using convergent-beam electron diffraction may elucidate the origins of these features.

\section{Conclusion}

The crystallization kinetics of as-deposited a- $\mathrm{TiO}_{2}$ films on amorphous substrates were analyzed in terms of classical phase-transformation theory, assuming spontaneous, homogeneous grain nucleation throughout the film volume and isotropic grain growth. Several subsequent experiments are planned: (1) measurement of thermal activation of grain nucleation and grain growth, by studying variations with annealing temperature; (2) characterization of the crystallized grain structure, including defect character, preferential orientation, and dependence of growth velocity on crystallographic direction; (3) electron-diffraction analysis during crystallization using TEM in situ annealing.

\section{Acknowledgments}

This work was supported by the U.S. Department of Energy, project no. DOE DE-FG02-08ER64624, and by the National Science Foundation, project no. 0453216.

\section{References}

[1] M. C. Kao, H. Z. Chen, S. L. Young, C. Y. Kung, and C. C. Lin, "The effects of the thickness of $\mathrm{TiO}_{2}$ films on the performance of dye-sensitized solar cells," Thin Solid Films, vol. 517, no. 17, pp. 5096-5099, 2009.

[2] K. V. Baiju, S. Shukla, K. S. Sandhya, J. James, and K. G. K. Warrier, "Photocatalytic activity of sol-gel-derived nanocrystalline titania," Journal of Physical Chemistry C, vol. 111, no. 21, pp. 7612-7622, 2007.

[3] S.-D. Mo and W. Y. Ching, "Electronic and optical properties of three phases of titanium dioxide: rutile, anatase, and brookite," Physical Review B, vol. 51, no. 19, pp. 13023-13032, 1995.

[4] I. N. Martyanov and K. J. Klabunde, "Comparative study of $\mathrm{TiO}_{2}$ particles in powder form and as a thin nanostructured film on quartz," Journal of Catalysis, vol. 225, no. 2, pp. 408416, 2004

[5] Y. U. Ahn, E. J. Kim, H. T. Kim, and S. H. Hahn, "Variation of structural and optical properties of sol-gel $\mathrm{TiO}_{2}$ thin films with catalyst concentration and calcination temperature," Materials Letters, vol. 57, no. 30, pp. 4660-4666, 2003.
[6] K. Bange, C. R. Ottermann, O. Anderson, U. Jeschkowski, M. Laube, and R. Feile, "Investigations of $\mathrm{TiO}_{2}$ films deposited by different techniques," Thin Solid Films, vol. 197, no. 1-2, pp. 279-285, 1991.

[7] B. K. Vainshtein, Fundamentals of Crystals, Springer, London, UK, 2nd edition, 1994.

[8] G. J. Exarhos and M. Aloi, "Crystallite growth kinetics in isothermally annealed sol-gel films," Thin Solid Films, vol. 193194, pp. 42-50, 1990.

[9] L. S. Hsu, R. Rujkorakarn, J. R. Sites, and C. Y. She, "Thermally induced crystallization of amorphous-titania films," Journal of Applied Physics, vol. 59, no. 10, pp. 3475-3480, 1986.

[10] M. Shiojiri, "Crystallization of amorphous titanium dioxide films prepared by vacuum-evaporation," Journal of the Physical Society of Japan, vol. 21, pp. 335-345, 1966.

[11] M. Shiojiri, H. Morikawa, and E. Suito, "Cinematographic observation of crystallization process of amorphous titanium dioxide films," Journal of Electron Microscopy, vol. 17, pp. 1-6, 1968.

[12] P. Kern, C. Jäggi, I. Utke, V. Friedli, and J. Michler, "Local electron beam induced reduction and crystallization of amorphous titania films," Applied Physics Letters, vol. 89, no. 2, Article ID 021902, 3 pages, 2006.

[13] H. Zhang and J. F. Banfield, "Kinetics of crystallization and crystal growth of nanocrystalline anatase in nanometer-sized amorphous titania," Chemistry of Materials, vol. 14, no. 10, pp. 4145-4154, 2002.

[14] G. Madras and B. J. McCoy, "Kinetic model for transformation from nanosized amorphous $\mathrm{TiO}_{2}$ to anatase," Crystal Growth \& Design, vol. 7, no. 2, pp. 250-253, 2007.

[15] J. W. Christian, Theory of Transformation in Metals and Alloys, Pergamon Press, Oxford, UK, 2nd edition, 1965.

[16] R. B. Iverson and R. Reif, "Recrystallization of amorphized polycrystalline silicon films on $\mathrm{SiO}_{2}$ : temperature dependence of the crystallization parameters," Journal of Applied Physics, vol. 62, pp. 1675-1681, 1987.

[17] R. Asahi, Y. Taga, W. Mannstadt, and A. J. Freeman, "Electronic and optical properties of anatase $\mathrm{TiO}_{2}$," Physical Review $B$, vol. 61, no. 11, pp. 7459-7465, 2000.

[18] P.-H. Jouneau and P. Stadelmann, EMS on Line, (C) 19951998, Centre Interdépartemental de Microscopie Electronique, EPFL, Lausanne, Switzerland. 

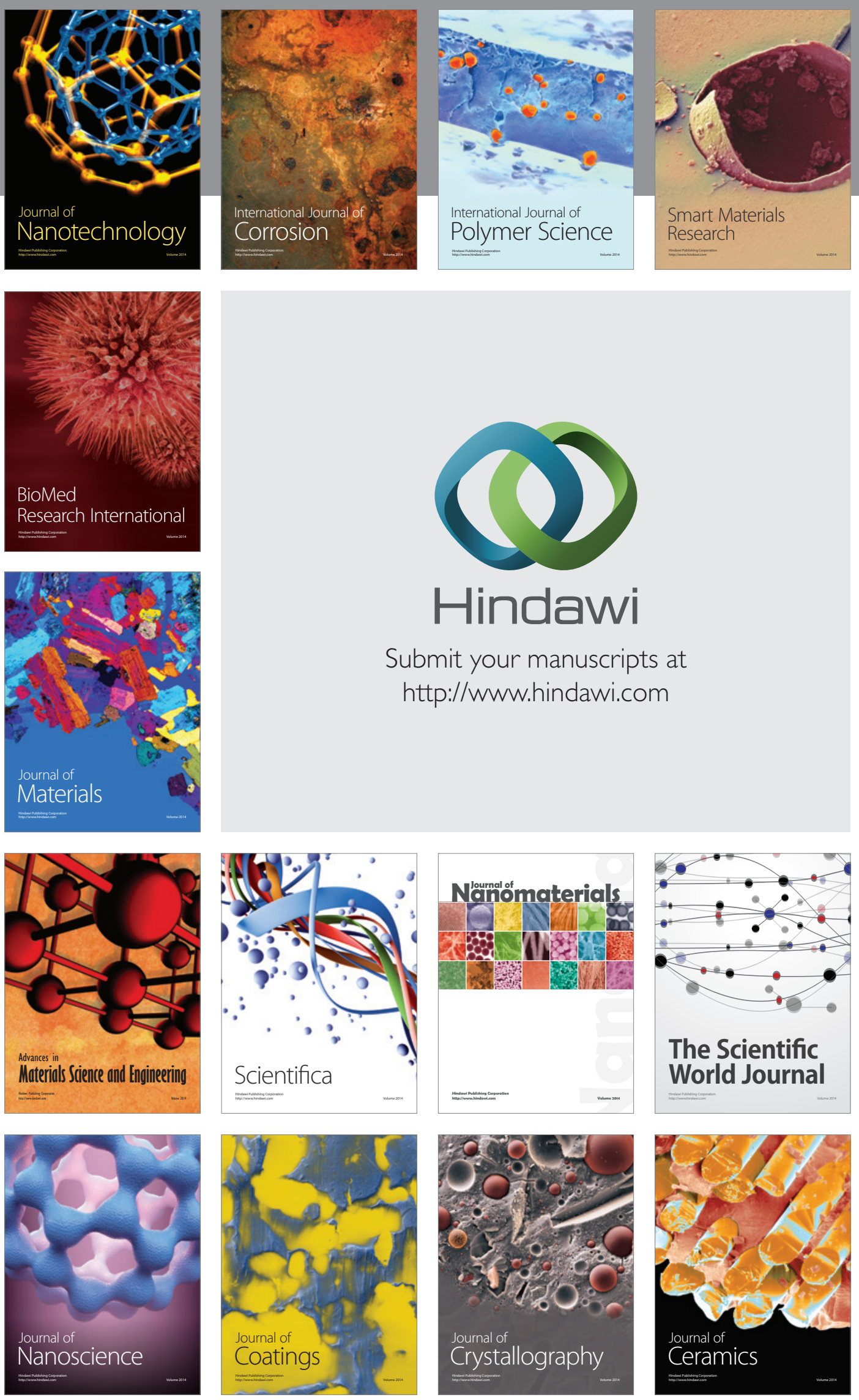

The Scientific World Journal

Submit your manuscripts at

http://www.hindawi.com

\section{World Journal}

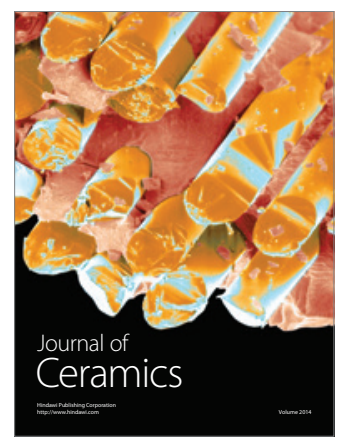

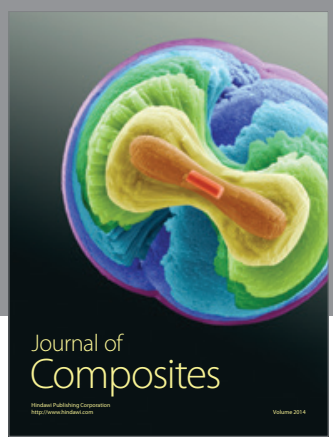
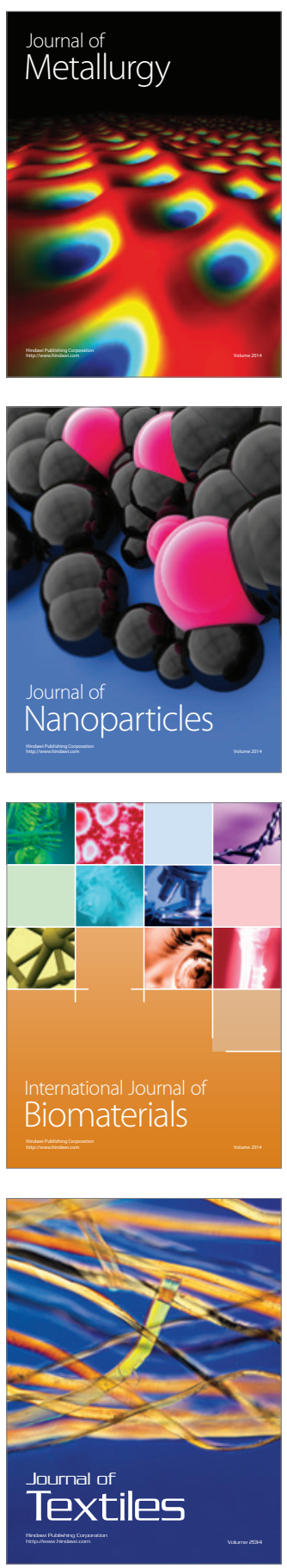